\title{
The small UAV longitudinal control law design based on genetic algorithms
}

\author{
Lin Feng ${ }^{1, a}$,Yuxi Wang ${ }^{2, b}$,Xiaoguang $\mathrm{Qu}^{3, \mathrm{c}}$ \\ ${ }^{1,2,3}$ School of automation, Shenyang Aerospace University, Shenyang, \\ 110136, China \\ aemail: Ifshenyang163@163.com, bemail:yuxi_wang11@163.com, \\ cemail:qxgshenyang163@163.com
}

Keywords: Design of Control law; Genetic Algorithms; PID control

\begin{abstract}
Small UAV autonomous flight control system is the core part of it, so the design of the control law under the flight control system is especially important.UAV control law design can be divided into longitudinal control law design and horizontal lateral control law design.This design is mainly aimed at the longitudinal control system, study the structure and control scheme.Design the longitudinal pitch attitude control law and highly keep modal control law. Finally in Angle of pitching control, for example, designed the PID parameters based on genetic algorithm to choose optimal control, by comparing the simulation, verify the feasibility of the algorithm.
\end{abstract}

\section{Introduction}

Small UAV has important application value in military and civil field. The flight control system is the core part of the UAV, accurate and stable control law is UAV flight safety and perform the task requirements. So more perfect control law design become the focus of current research.

At present, the PID control [1] in UAV control strategy is one of the more widely applied. But its parameter setting has been a very difficult problem. And small UAV control system is very complex, increase of uncertainty, but require higher performance index. The conventional PID control method can't completely meet the UAV [2][3] control law design. PID control strategy based on genetic algorithm is proposed in this paper. Its structure is simple, strong robustness, is an efficient optimization method. But the traditional genetic algorithm prone to premature and slow convergence speed.

Based on the small UAV longitudinal control law design in this paper, for example, using the improved genetic algorithm for PID parameters selection. Obtained is better than traditional genetic algorithm optimization results and the parameters of the faster convergence speed. Finally through MATLAB [4] simulation shows that the algorithm is effective can be applied to small UAV control law design.

\section{Genetic algorithm introduction and features}

Genetic algorithm [5] simulates the genetic and evolutionary process of organisms in the natural environment and form a kind of a global optimization probability search algorithm. It first put forward by the United States professor at the university of Michigan, originated in the $1960 \mathrm{~s}$, the study of natural and artificial adaptive systems. $1970 \mathrm{~s}$ De Jong based on the idea of genetic algorithm on the computer to do a lot of pure numerical function optimization experiment. On the basis of a series of research work. Goldberg were summarized in the 1980s, has formed the basic framework of genetic algorithm.

Genetic algorithm [6] is a natural genetics and computer science of mutual infiltration and combination of new calculation method. Genetic algorithms as a new global optimization search algorithm, its simple common, has strong robustness, is suitable for the complex system optimization of parallel processing operations. On the other hand, compared with other algorithms, 
the robustness of genetic algorithm and will make the effect of parameters on the search results will be as low as possible.The standard genetic algorithm is a "generate and test" search algorithm of iterative process, Its basic process flow shown in Figure 1.

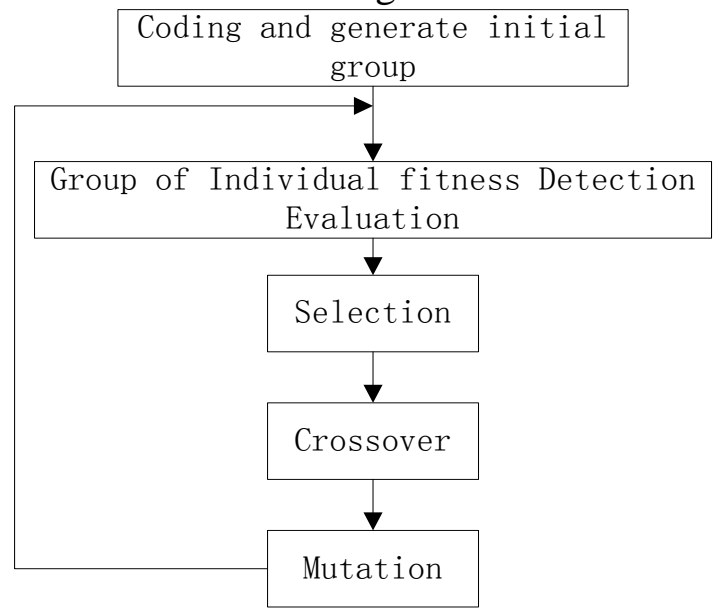

Figure 1 The basic flow of the standard genetic algorithm

As shown in figure shows. Genetic algorithm is a group of type operation, the operation to all individuals in the group as an object, selection, crossover, mutation, are the three main operating operator of Genetic algorithm. They form the so-called genetic operation. Genetic algorithm has the characteristics of the other traditional algorithms have not. Currently, Genetic algorithms have been widely used in PID control. Because the genetic algorithm itself has a strong global optimization capability and robustness characteristics, therefore suitable for complex nonlinear control systems. This article is presented on the basis of a genetic algorithm based on PID parameter optimization algorithm, and the algorithm was applied to small UAV control law design, by MATLAB simulation[7][8], satisfactory results were achieved.

\section{Longitudinal motion control law design}

Small unmanned aircraft longitudinal motion refers to the aircraft motion in the symmetry plane, mainly includes the aircraft's longitudinal Angle motion and vertical line movement. The aircraft's longitudinal Angle motion is the pitch Angle movement of aircraft, the aircraft's longitudinal Angle motion is mainly through the elevator or flat tail to control. The aircraft's longitudinal line movement refers to the height of the aircraft under the action of force.

\section{The stability of the pitching Angle and control}

Angle of small unmanned aircraft longitudinal motion control is mainly control the pitching Angle of the plane, pitching Angle control law is the most basic control law of the longitudinal control law, its main function is to stabilize the flight attitude. The control method is generally PID control.Pitch attitude a modal plane can be keep in a given pitch reference posture $\theta_{\text {ref }}$, it was established by the flight control computer, control system turned on after trying to maintain this stance. The principle of pitching Angle control system structure diagram as shown in figure 2.

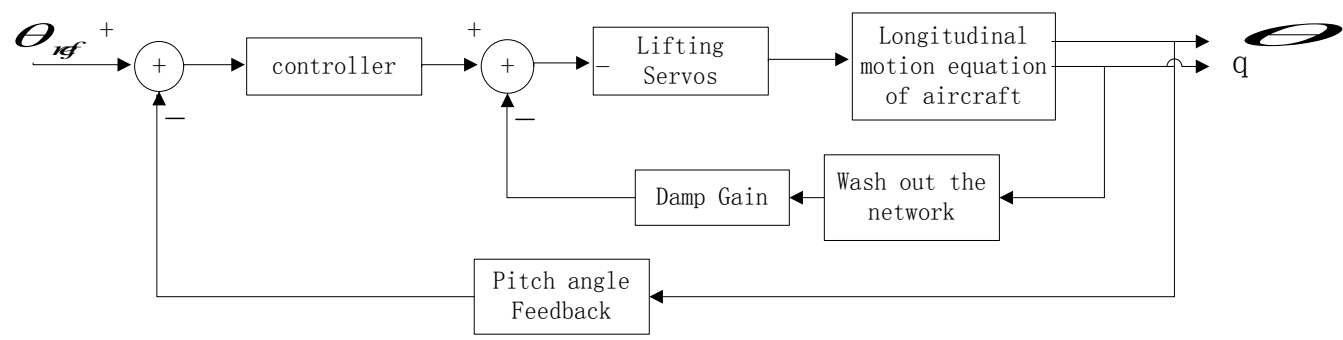

Figure 2 Pitch angle control chart

As can be seen from the structure diagram. In order to effectively restrain and reduce the 
interference, usually introduce pitching Angle rate to the inner loop of a control system, pitching Angle as outer loop to control the longitudinal angular motion. The introduction of inner loop in the pitch rate feedback is equivalent to change the longitudinal damping derivative of UAV , increased its longitudinal damping, to make it short cycle of modal damping characteristics were improved.Outer loop constitutes the pitching Angle stability loop, can improve the damping characteristics of UAV.

The control law can be expressed as: $\quad \delta_{e}=K_{e}^{\Delta \theta}\left(\theta-\theta_{\text {ref }}\right)+K_{e}^{Q} Q$

Among them, $\theta_{r e f}$ is the target pitching Angle, $\theta$ is the actual pitching Angle, $q$ is pitching Angle speed, $\delta_{e}$ is the elevator output, $K_{e}$ represents PID controller.

The formula is: $K_{e}=K_{p}+K_{i} / s+K_{d} \cdot s$

$K_{p}, K_{i}, K_{d}$ is proportional gain, integral gain, differential Gain. $s$ is the Laplace operator.

\section{The stability of the flight altitude and control}

Altitude stability and control plays an important role in UAV flight.UAV longitudinal control is mainly plane height control. Because of interference Angle stability system, such as the presence of vertical wind and other interference static attitude angle difference, thus resulting in a high degree of drift, it can not be directly applied to the flying height of stabilization and control system. Therefore, in the flying height of stability and control system need to direct measurement of the flight altitude, using the height difference sensor, such as barometric altimeter or Radio altimeter. According to the height difference of information to direct control of aircraft flight attitude. Because of the height and pitch Angle of aircraft has a coupling relationship. The height deviation signal input pitching Angle control system, control the attitude of the aircraft, change the aircraft's flight path Angle, control aircraft movements, until the height difference is zero, make the plane back to reserve level. Maintain control system principle block diagram is shown in figure 3.

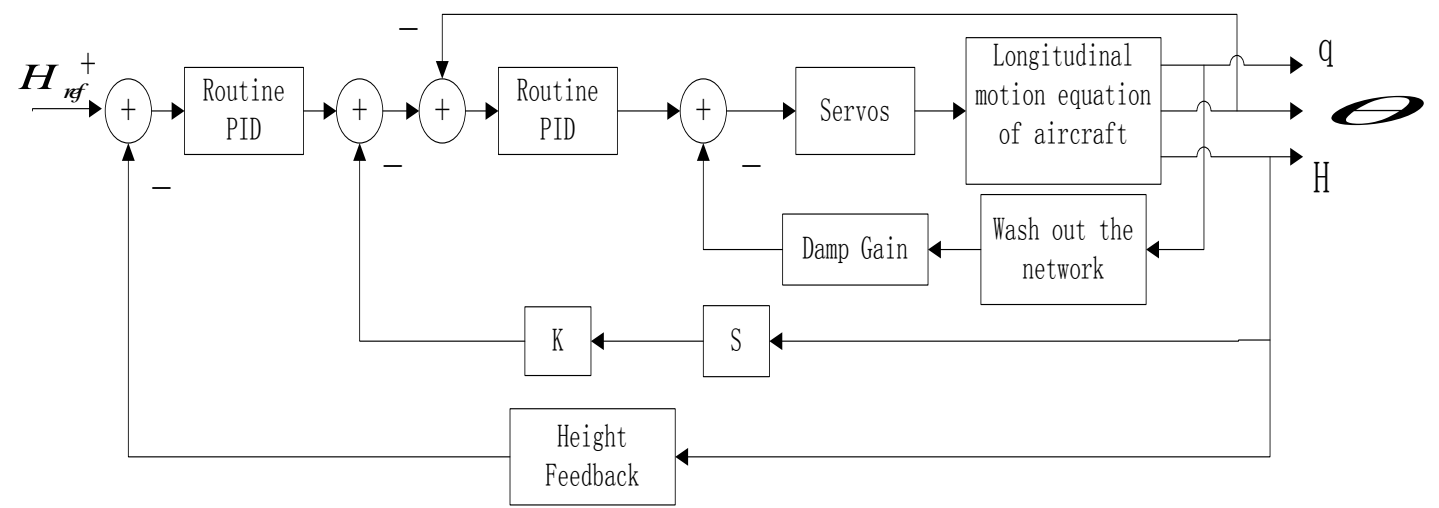

Figure 3 Height control system chart

From the above chart we can know, the inner loop of the pitch angle control system. Altitude hold control laws Design based on it, it has been designed without changing the attitude angle control system. Usually assumes that the airplane pitching Angle to a stable value.Greater than this value, the plane climbed; Less than this value, the plane fell; Equal to the value, aircraft altitude hold.

The control law can be expressed as: $\quad \delta_{e}=K_{e}^{\theta} \theta+K_{e}^{Q} Q+K_{e}^{\Delta H} \Delta H+K_{e}^{H} H$

Among them, $\Delta H$ is relative to a given height deviation,

$$
\Delta H=H-H_{\text {ref }}
$$

$H_{\text {ref }}$ is the target height, $H$ is the actual height. Visible, control law is mainly based on the pitch angle stabilization loop configuration, in order to avoid the fluctuation appeared oscillation in a given height, height difference should be introduced first-order differential signal, to improve the damping characteristics of highly stable system. 


\section{Test results}

\section{To pitch angle as an example}

Longitudinal stability augmentation control system makes the aircraft's longitudinal stability, maneuverability, and mobility are improved effectively. Stability augmentation system make the system of closed loop poles with the original open-loop poles have obvious differences of the plane, longitudinal attitude control system is a good inside the loop, make the control system has good stability, response and control precision[9]. To pitch angle as an example, you can use the Matlab's simulink toolbox, on aircraft linearized state equation module as a control object longitudinal movement, preliminary design, and then to actual control system, as longitudinal movement of the inner loop, simulation is carried out, calculation and parameter adjustment work.

Linearized equation of state is:

$$
\left[\begin{array}{c}
\dot{v} \\
\dot{\alpha} \\
\ddot{\theta} \\
\dot{\theta} \\
\dot{h}
\end{array}\right]=\left[\begin{array}{ccccc}
-n_{1 v} & -n_{1 \alpha} & 0 & -n_{1 \theta} & 0 \\
-n_{2 v} & -n_{2 \alpha} & 1 & -n_{2 \theta} & 0 \\
-N_{3 v} & -N_{3 \alpha} & -N_{3 \theta} & -N_{3 \theta} & 0 \\
0 & 0 & 1 & 0 & 0 \\
n_{4 v} & -n_{4 a} & 0 & n_{4 \theta} & 0
\end{array}\right]+\left[\begin{array}{cc}
0 & 0 \\
0 & -n_{2 \delta_{z}} \\
0 & -n_{3 \delta_{z}} \\
0 & 0 \\
0 & 0
\end{array}\right] \cdot\left[\begin{array}{l}
\delta_{p} \\
\delta_{z}
\end{array}\right]
$$

Has a point in state space pitch channel is expressed as follows:

$$
\begin{aligned}
& A=\left[\begin{array}{ccccc}
-0.0465 & -0.2367 & -0.1710 & 0 & 0 \\
-0.0069 & -0.2764 & 0.0001 & 1 & 0 \\
0 & 0 & 0 & 1 & 0 \\
-0.7696 & -5.1955 & 0 & -0.2752 & 0 \\
0.0032 & -4.1255 & 4.1255 & 0 & 0
\end{array}\right] \quad B=\left[\begin{array}{c}
0 \\
-0.1638 \\
0 \\
-6.6520 \\
0
\end{array}\right] \\
& C=\left[\begin{array}{lllll}
0 & 0 & 1 & 0 & 0 \\
0 & 0 & 0 & 1 & 0
\end{array}\right] \quad B=\left[\begin{array}{l}
0 \\
0
\end{array}\right]
\end{aligned}
$$

The result can be obtained by the space Equation:

$$
\frac{\theta(s)}{\delta_{z}(s)}=\frac{-171.1 s+360.6}{s^{3}+13.981 s^{2}+66.28 s+26.7}
$$

Using Matlab's simulink simulation toolbox,the pitch angle of the step response shown in Figure4.

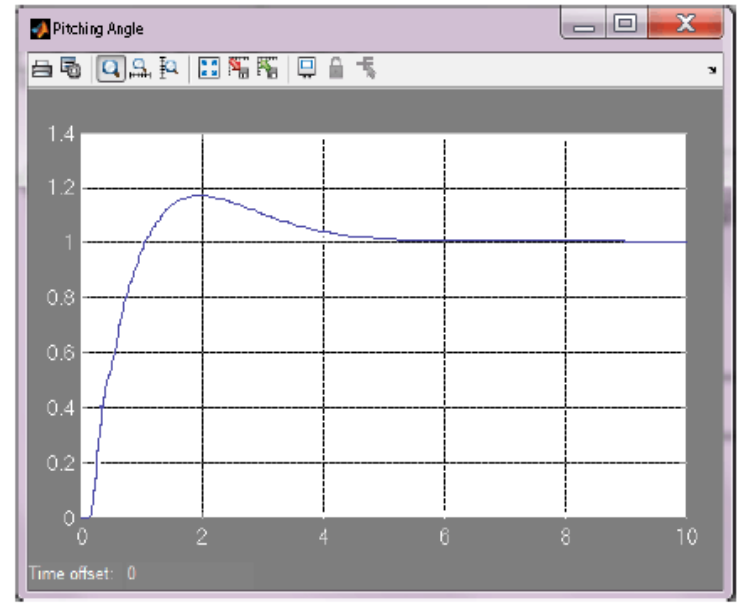

Figure 4 The pitch angle of the step response

\section{PID control law is designed based on genetic algorithm}

PID has very important applications in small UAV Control, in order to meet the system performance indicators. This article selects the genetic algorithm for PID parameters optimization, aking into account the dynamic performance of the system, Steady performance and stability: Quick response(adjustment time is short); Steady-state high precision(no steady-state error);Good dynamic performance(response no overshoot and no oscillation). 
PID Control Based on Genetic Algorithms, it is the case of the known control strategy.

$$
u_{t}=K_{p} e(t)+K_{D} d_{e(t)} / d_{t}+K_{I} \int e(t) d_{t}
$$

Using a genetic algorithm for PID controller parameters $\left.\mid K_{p}, K_{I}, K_{D}\right\rfloor$ optimum choice.Finding the right set of control parameters allow. Make a given performance indicators for the best.

$$
J=\max \left\{g\left(K_{P}, K_{I}, K_{D}\right)\right\}
$$

This method is a kind of don't need any initial information and can seek the global optimal solution and efficient method of optimum combination. To achieve this optimization design block diagram shown in Figure 5.

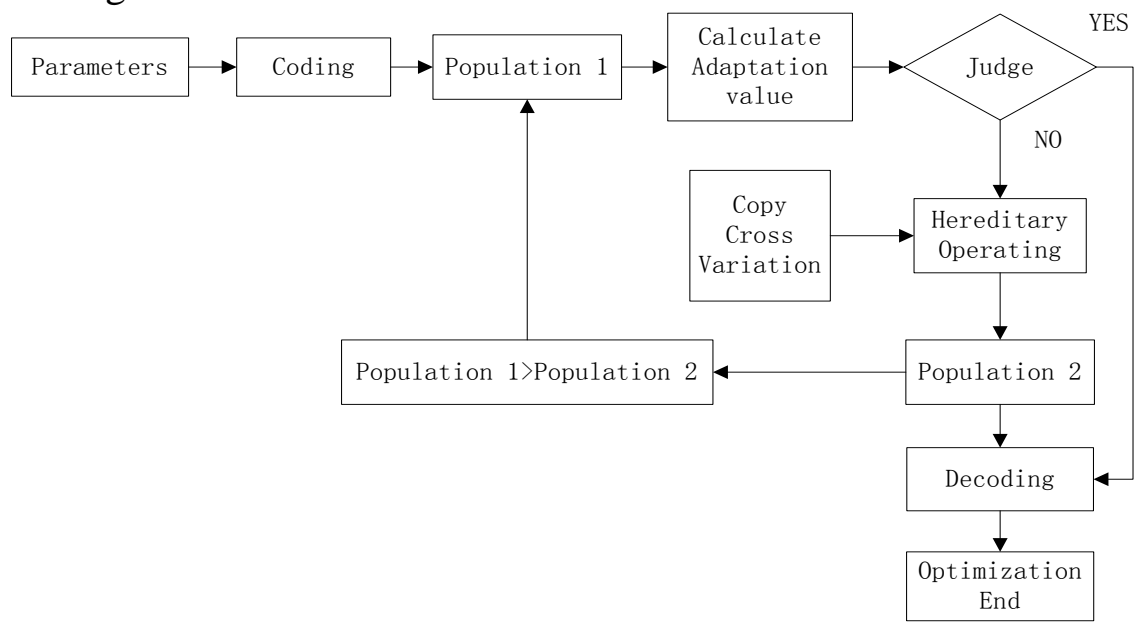

Figure 5 The flow chart of genetic algorithm

Genetic algorithm is adopted to improve the setting of PID coefficient of three has the advantage of initial condition is not so harsh,simplicity of operator, optimization speed fast, it is a global optimization algorithm. Take the objective function for:

$$
J=\int_{0}^{\infty}\left(w_{1}|e(t)|+w_{2} u^{2}(t)\right) d_{t}+w_{3} \cdot t_{s}
$$

In order to avoid overshoot, use Punitive function, once produce overshoot, overshoot volume as one of the most excellent indicators, at this point the best indicators as follows:

$$
e(t)<0 \quad J=\int_{0}^{\infty}\left(w_{1}|e(t)|+w_{2} u^{2}(t)+w_{4}|e y(t)|\right) d_{t}+w_{3} \cdot t_{s}
$$

Among them, $w_{1}, w_{2}, w_{3}, w_{4}$ is the weighted value, and $w_{4}>w_{3}, e(t)$ is the system error, $y(t)$ is the system output, $u(t)$ is to control the output, $t_{s}$ is the rise time.The scope of PID is [0,20],fetch $w_{1}=0.999, w_{2}=0.001, w_{3}=2.0, w_{4}=100$.

Among them $\quad e y(t)=y(t)-y(t-1)$

The fitness function can now take as follows:

$$
f=\frac{1}{J}=\frac{1}{\int_{0}^{\infty}\left(w_{1}|e(t)|+w_{2} u^{2}(t)+w_{4}|e y(t)|\right) d_{t}+w_{3} \cdot t_{s}}
$$

We use gray coding, and randomly generated initial population size is 30 . Because have in the system $K_{P}, K_{I}, K_{D}$ three parameters to be optimized, these three parameters are components form a three-dimensional vector, and coding.

\section{Simulation results}

We use this algorithm for pitch Angle control law of the PID parameters selection, after 100 generations of optimization. Into the process of $\mathrm{J}$ function optimization and the PID control based on genetic algorithm after setting pitching Angle step response as shown in figure 6 and figure 7. 


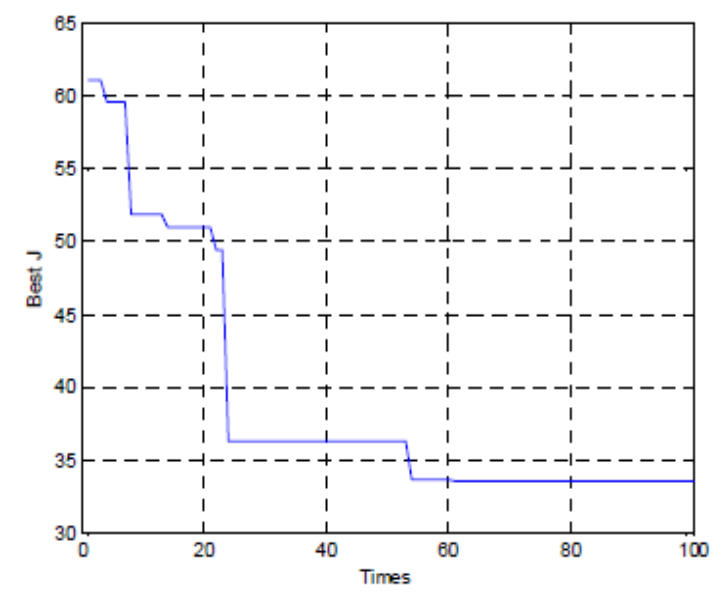

Figure 6 Into the process of $\mathrm{J}$ function optimization

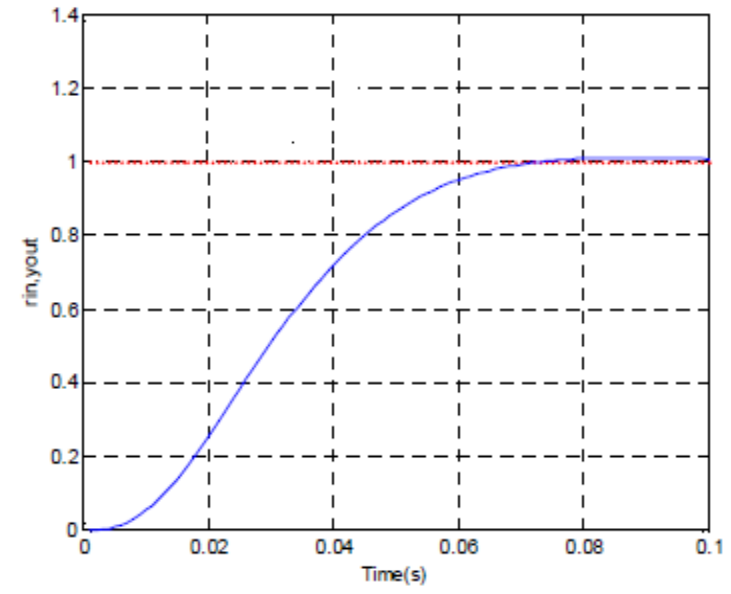

Figure 7 After step response tuning

By above can know, Figure 5 pitching Angle is obtained by linearized longitudinal motion equation of step response has obvious overshoot, and slow convergence time. Figure 6 and figure 7 is given a more intuitive using genetic algorithm to choose the three coefficients of PID control, by optimizing the objective function to obtain the pitch angle step response curve, to observe the application of the improved algorithm has faster convergence, without overshoot. The algorithm largely improve the performance of the UAV control law, to prove the feasibility and effectiveness of the algorithm.

\section{Conclusion}

Due to the limited space in this paper, only the pitch angle Case. By improved genetic algorithm for PID parameters selection, to realize the rapid response, high steady precision and good dynamic performance indicators, its highly maintain control can be used the same method.

\section{References}

[1] Yucheng Li.The genetic algorithm in the application of PID control[D].North China University of Technology.2006

[2] Sengtang Wu,Yuhua Fei.Flight Control System[M].BeiJing:Beijing University of Aeronautics and Astronautics Press,2005:98-103

[3] Kaibo Bi,Xiaodong Wang, Zhiping Liu.Vehicle Guidance and Control and Matlab simulation technology[M].BeiJing:National Defense Industry Press,2008:98-150

[4] Haiquan Xiong.Aircraft Flight Dynamics [M].BeiJing:Aviation Industry Press,1990:22-34

[5]Guoliang Chen,Zhenquan Zhuang,Dongsheng Wang.Genetic Algorithm and Its Application[M].BeiJing:People's posts and telecommunications press ,1999:5-22

[6] Zhimin Yang,Wang XU,Xianyi Zhuang.Genetic algorithm is applied in the field of automatic control were reviewed[J].Information and Control,2000(4)330-338

[7]Wen Xin,Zhou Lu,Danli Wang,Xiaoying Xiong.MATLAB neural network application design[M].BeiJing:Science Press,2001:207-243

[8]Suqing Wang,Weifu Jiang.Based on MATLAB / SIMULINK of PID parameter tuning[J].Techniques of Automation and Applications,2009(3)24-28

[9] LinkensD A,NyongesaH O.Genetic algorithms for fuzzy control.IEE Proc Control Theory Appl.1995 